\title{
A Further Study of the Quantitative Evaluation of the Fine Amorphous Structure Distribution of a Semi-Crystalline Polymer Solid from the Dynamic Loss Tangent $(\tan \delta)$ vs. Temperature Curve
}

\author{
Sei-ichi MANABE and Kenji KamIDE \\ Textile Research Laboratory, Asahi Chemical Industry Co., Ltd., \\ Hacchonawate 11-7, Takatsuki, Osaka, Japan
}

(Received December 5, 1983)

\begin{abstract}
An attempt was made to improve the method (MKN method) for evaluating the fine structure of the amorphous region in a semi-crystalline polymer solid from the dynamic loss tangent $(\tan \delta) v s$. temperature $(T)$ curve, originally proposed by the authors, taking into account the contribution of crystalline region acting in series against an external force. The volume fraction of the segments contributing to the $\alpha_{a}$ dispersion, and the apparent activation energy for $\alpha_{a}$ dispersion in addition to the $\tan \delta v s$. T curve are prerequisite to the new method (MK method). This method affords some structural parameters $\lambda$ and $\phi$ defined in Takayanagi's model with which the amorphous region works in parallel and in series against an external force, and the distribution function of a packing density of polymer molecules in an amorphous region.
\end{abstract}

KEY WORDS Dynamic Loss Tangent / Takayanagi's Model / Fine

Structure / Amorphous Region / Crystalline Polymer / Viscoelasticity /

Nylon 66 /

Many physical properties of a polymer solid, including tensile modulus, ultimate strength and elongation, are influenced by its moulding or forming conditions. This strongly suggests that a super molecular rather than the molecular structure remarkably influences the physical properties of the polymer solid. First, we define the second-order structure, a kind of super molecular structure, as follow: This structure is characterized by the aggregation state of a polymer chain in a hypothetical cubic body with one side about $10 \mathrm{~nm}$ in length. An accurate determination of the length of this body depends on the molecular structure (i.e., the first-order structure) of the polymers. The structural parameters characterizing the second-order structure are, for example, crystal lattice constant, crystal size, orientation of the crystals and degree of crystallinity in the crystalline phase and packing density and regularity in aggregation of the polymer chain in non-crystalline phase.

Up to now, the first- and the second-order structural parameters have been extensively studied, but the following experimental facts indicate the necessity for identifying higher order structures than those of a second order for the semi-crystalline polymer solid: (1) The degree of crystallinity of the semi-crystalline polymer samples varies greatly, depending on the methods adopted for determination. (2) The dyability, hygroscopicity and chemical reactivity of the semi-crystalline polymer solid cannot be correlated quantitatively with first and second order structures.

Since the second order structures in a sample are not all the same with respect to the aggregation state of polymer chains, a kind of distribution of the second order structure should be taken into consideration. The halfwidth values of the experimental dynamic loss tangent $(\tan \delta)$-temperature $(T)$ curves in the 
primary dispersion ( $\alpha_{a}$ dispersion) region of the semi-crystalline polymers are more than twice the theoretical values calculated by the theory derived by Manabe-KamideNakayama (simple MKN theory ${ }^{1}$ ) based on the Rouse, Tobolsky and Aklonis (RTA) theory. ${ }^{2,3}$ In this theory, the homogeneous packing state of a polymer chain in the second order structure is assumed. Manabe, Kamide, and Nakayama ${ }^{1}$ gave a theoretical background for analyzing $\tan \delta-T$ curve in the temperature range of the $\alpha_{a}$ dispersion. This is a generalized simple MKN theory taking into account the distribution of the packing density of the polymer chain in an amorphous region so as to represent the variety of second order structures in the sample (referred to as MKN theory). They successfully evaluated the average and distribution of the molecular packing density, applying the MKN theory to the experimental $\tan \delta-T$ curve.

The main reasons for using the $\tan \delta-T$ curve rather than that of the loss modulus $E^{\prime \prime}-$ $T$ are as follows: (1) Measurement of $\tan \delta$ can be made with greater precision than that of $E^{\prime \prime}$ and $\tan \delta$ is independent of the dimensions of the sample to be measured; (2) the theoretical $\tan \delta-T$ curve for the homogeneous amorphous region idealized can be approximated by an equilateral triangular shape, ${ }^{3}$ and (3) the numerical calculations for $\tan \delta-T$ curve show that additivity in $\tan \delta$ holds approximately when using the elastic modulus fraction of the component to that of the whole system when all components constituting the system act in parallel for the external force. ${ }^{4}$ From a practical standpoint of view, the merits of article (1) are very valuable since change in the dimensions of the sample during isochronal measurement is unavoidable, especially in the case of a sample consisting of a bundle of fibers, some fixed loosely and some tightly.

It should be noted that the MKN theory does not consider a higher order structure than that of a second order. Takayanagi ${ }^{4}$ was the first to point out that the dynamic viscoelasti- cities of a semi-crystalline polymer solid are sensitively influenced by the mixing state of crystalline and non-crystalline regions. $\mathrm{He}$ proposed a "Takayanagi's model" as a dynamic equivalent model, in which the heterogeneous aggregation of polymer chains in an amorphous region can be ignored. This model may be regarded as a third order structural model representing the texture of crystalline polymers with crystalline and non-crystalline regions.

This article presents an improved MKN theory for application to a system with heterogeneity in mixed crystalline and noncrystalline regions. We also examine to what extent the MKN theory can be applied to semi-crystalline polymers.

\section{THEORETICAL BACKGROUND}

Figure 1 shows schematic representations of fine polymer solid structures and the dynamically equivalent model (Takayanagi's model). The polymer solid was divided into numerous cubic bodies about $10 \mathrm{~nm}$ on a side (i.e., the 2nd-order structural unit) and was assumed to be an aggregate of 2 nd-order elements (Figure 1b).

Within a given 2nd-order element, the aggregation of polymer chains can be regarded as uniform from the standpoint of segmental microbrownian movement giving rise to the $\alpha_{a}$ dispersion. The packing density was primarily correlated to the free volume fraction of the element and thus reflects the glass transition temperature of the 2 nd-order element on the basis of the iso-free volume condition of the glass transition. The packing density was characterized by the peak temperature $T_{\max }$ of $\tan \delta-T$ curve related to the $\alpha_{a}$ dispersion; i.e., an element whose polymer chains are loosely packed, has a lower $T_{\max }$ than one with closely packed chains. This can be seen from Figure lc.

The 2nd-order structural elements for a given polymer solid sample are separated from 


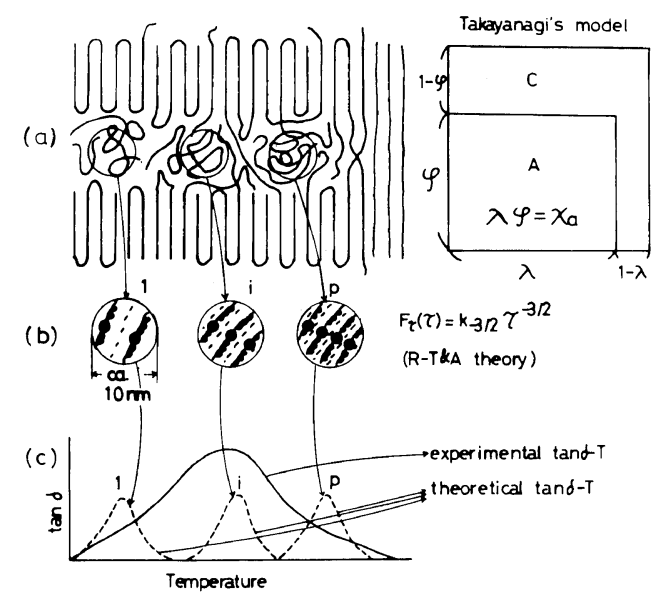

Figure 1. Schematic representation of the fine structure of an amorphous region and the dynamically equivalent model of this structure (Takayanagi's model), and the $\tan \delta-T$ curve in the temperature region of $\alpha_{a}$ absorption: (a), a fine structure and its dynamically equivalent model (Takayanagi's model). The line indicates a polymer chain; (b), spring and bead model representing the viscoelasticity of a 2 nd order element in an amorphous region. The relaxation time distribution $H_{\tau}(\tau)$ is given by the RTA theory; (c) $\tan \delta-T$ curves of the elements $1, i$, and $p$ and an experimental $\tan \delta-T$ curve.

each other and arranged in the order of increasing packing density, numbering from $i=1$ to $p$ for all elements. In this case, even the elements, which show no peak in the $\alpha_{a} \cdot$ dispersion region (i.e., the elements corresponding to the crystalline and non-crystalline/nonamorphous regions) are included for comparison as an extreme case. Here, the amorphous region is defined as the region in which segments show significant peaks at their $\alpha_{a}$ dispersions, and the crystalline region as the region confirmed as crystalline by the $\mathrm{X}$-ray diffraction method. There remains the region that is neither crystalline nor amorphous. In general, the elements with different $i$ values are different in their packing densities. In Figure $2 \mathrm{a}$, the packing density of the $i$-th element is the same as those of $i+1, i+2, \cdots, i+j$-th elements, as a special case. The model in Figure 2a can be expressed as a parallel model of amorphous, non-amorphous/non-crys- (a)

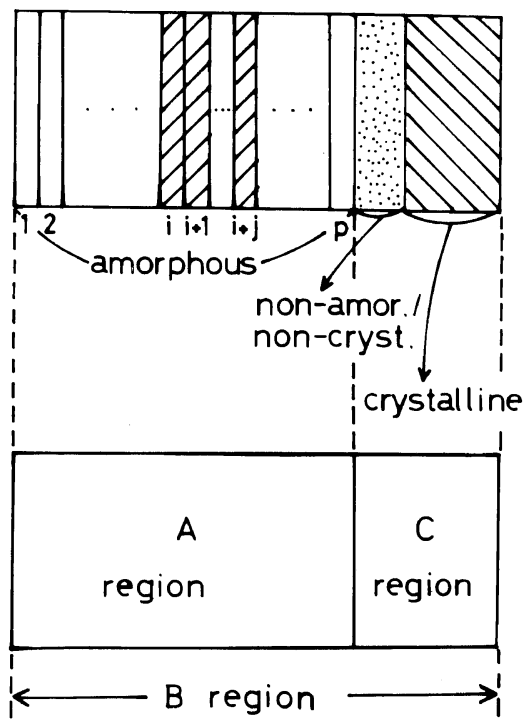

Figure 2. Schematic representation of the fine structure in a crystalline polymer solid of parallel portion (B region) constructed with amorphous, non-crystalline/ non-amorphous, and crystalline regions: Region $\mathrm{A}$, amorphous region; region $\mathrm{C}$, crystalline and noncrystalline/non-amorphous regions; region $\mathrm{B}$, region combining the $\mathrm{C}$ and $\mathrm{A}$ regions in a parallel manner.

talline, and crystalline regions. The portion whose dynamically equivalent model is expressed by a parallel combination of the $\mathrm{C}$ and $\mathrm{A}$ (amorphous) regions as shown in Figure $2 \mathrm{a}$, was termed the $\mathrm{B}$ region. The MKN theory can be applied to this region since both $\mathrm{A}$ and $\mathrm{C}$ act in parallel for the external force. Figure 3 illustrates the improved MKN theory (referred to as MK theory), compared with Takayanagi's model and the MKN theory. The non-crystalline region in Takayanagi's model is divided into many elements of $A_{1}$ to $\mathrm{A}_{p}$, as in the $\mathrm{MK}$ theory (Figure 3a). The apparent activation energies for the $\alpha_{a}$ dispersion of the elements $A_{1}, A_{2}, \cdots$, and $A_{p}$ differ from each other. The viscoelastic properties of the structural model in Figure 3a can be represented by the two mechanical models shown in Figure $3 \mathrm{~b}$. These two models are equivalent with respect to viscoelasticity. ${ }^{5}$ Model I can be translated into the model II by eq 1 and 2, (see Appendix I). 


$$
\lambda_{\mathrm{I}}=\left[\left(1+\chi_{a}\right) \lambda_{\mathrm{II}}-\chi_{a}^{2}\right] / \lambda_{\mathrm{II}} \chi_{a}
$$

and

$$
\tan \delta_{\mathrm{I}}=\tan \delta_{\mathrm{II}}
$$

where suffixes I and II refer to models I and II, respectively, and $\lambda$ is the parameter expressing the fraction of the amorphous region acting in parallel to an external force as shown in Figure 3. Model I was modified by introducing $\mathbf{A}_{i}$ which represents the distribution of the packing density of a polymer chain in the amorphous region in the A region as shown in Figure $3 \mathrm{~b}$. The $\mathrm{MKN}$ theory can be applied only to the $\mathrm{B}$ region. The viscoelastic properties of this region are shown by the dynamically equivalent model of Figure $3 c .^{1}$

\section{Derivation of the Analysis Method of Tan $\delta_{\mathrm{b}}-$ \\ T Curve; MKN Method ${ }^{1}$}

The mechanical behavior of a 2nd-order structural element $\left(A_{i}\right.$ in Figure $\left.3 b\right)$ can be expressed by a generalized Maxwell model (Figure 3c). In Figure 3c, the $j$-th polymer segment in the $i$-th element behaves as a Maxwell model with relaxation time, $\tau_{j i} . N$ is the total number of polymer segments in the element and is expected to vary with the packing density of the polymer segment. $N$ also corresponds to the number of relaxation times of a 2 nd-order element. Tan $\delta$ for the $i$-th 2nd-order structural element, $\tan \delta_{i}$, is expressed by

$$
\tan \delta_{i}=\frac{\sum_{j=1}^{N} \frac{\omega \tau_{j i}}{1+\omega^{2} \tau_{j i}^{2}}}{\sum_{j=1}^{N} \frac{\omega^{2} \tau_{j i}^{2}}{1+\omega^{2} \tau_{j i}^{2}}+a^{-1}}
$$

where $\tau_{j i}$ is the relaxation time of the $j$-th Maxwell model of $i$-th element, $\omega$, the measured angular frequency $\left(\mathrm{rad} \mathrm{s}^{-1}\right)$, and $a$, the modulus ratio of $E / E_{0} . E$ is the modulus of the Maxwell model and $E_{0}$, that following the $\alpha_{a}$ dispersion of the element. These moduli are common to all the elements in Figure 3.

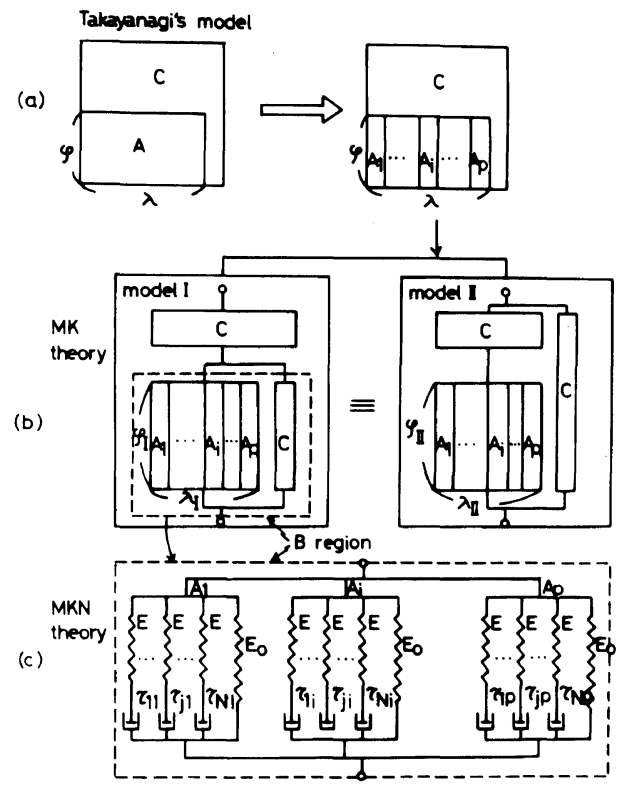

Figure 3. Illustration of the MK theory compared to Takayanagi's model: (a), Takayanagi's model and that adopted in the MK theory; (b), dynamically equivalent models of models I and II in the MK theory; (c), detailed discription of the dynamically equivalent model of the B region given by the dashed orthogonal region in Figure $3 \mathrm{~b}$.

$\tau_{j i}$ is given by Rouse, Tobolsky, and Aklonis (RTA) theory, assuming an isolated chain molecule in the homogeneous environment of the intermolecular interaction as follows:

$$
\tau_{j i}=a_{0}^{2} N^{2} \xi_{0 i} / 6 \pi^{2} j^{2} k T
$$

where, $a_{0}$ is the root mean square end-to-end distance of a segment $\xi_{0 i}$, the friction coefficient of the segment of the $i$-th 2 nd order structural element and $k$ is the Boltzman constant.

In general, the viscoelastic behavior of the element is very insensitive ${ }^{5}$ to $N$ if $N>10^{3}$. When $N \gg 1$, eq 3 is approximated by eq 5 with high accuracy for all cases:

$$
\tan \delta_{i}=\frac{\int_{\tau_{1 i}}^{\tau_{u i}} \frac{\omega \tau}{1+\omega^{2} \tau^{2}} H(\tau)_{i} \mathrm{~d} \tau}{\int_{\tau_{1 i}}^{\tau_{u i}} \frac{\omega^{2} \tau^{2}}{1+\omega^{2} \tau^{2}} H(\tau)_{i} \mathrm{~d} \tau+a^{-1}}
$$


with

$$
\tau_{u i}=N^{2} \tau_{1 i}
$$

where, $H(\tau)_{i}$ is the distribution function of relaxation time of $i$-th 2 nd-order element, i.e., the number of the segments whose relaxation times are between $\tau$ and $\tau+\mathrm{d} \tau$, given by $H(\tau)_{i} \mathrm{~d} \tau, \tau_{u i}$ and $\tau_{1 i}$ are the maximum and minimum relaxation times of $i$-th 2 nd-order element, respectively. $H(\tau)_{i}$ is derived from the RTA theory assuming the continuous distribution of $\tau_{j i}$ to be as follow:

$$
\begin{aligned}
H(\tau)_{i} & =\mathrm{d}(N-j) / \mathrm{d} \tau_{j i} \\
& =(1 / 2)\left(a_{0} N / \pi\right)\left(\xi_{0 i} / 6 k T\right)^{1 / 2} \tau^{-3 / 2}
\end{aligned}
$$

By putting eq 6 and 7 into eq 5 we obtain

$$
\begin{aligned}
\tan \delta_{i}= & {\left[\operatorname { l n } \left\{\left[1+\left(2 \omega N^{2} \tau_{1 i}\right)^{1 / 2}+\omega N^{2} \tau_{1 i}\right]\right.\right.} \\
& \times\left[1-\left(2 \omega \tau_{1 i}\right)^{i / 2}+\omega \tau_{1 i}\right] \\
& \times\left[1-\left(2 \omega N^{2} \tau_{1 i}\right)^{1 / 2}+\omega N^{2} \tau_{1 i}\right] \\
& \left.\times\left[1+\left(2 \omega \tau_{1 i}\right)^{1 / 2}+\omega \tau_{1 i}\right]\right\} \\
& +2\left\{\tan ^{-1}\left[1+\left(2 \omega N^{2} \tau_{1 i}\right)^{1 / 2}\right]\right. \\
& -\tan ^{-1}\left[1+\left(2 \omega \tau_{1 i}\right)^{1 / 2}\right] \\
& +\tan ^{-1}\left[\left(2 \omega N^{2} \tau_{1 i}\right)^{1 / 2}-1\right] \\
& \left.\left.-\tan ^{-1}\left[\left(2 \omega \tau_{1 i}\right)^{1 / 2}-1\right]\right\}\right] \\
& \times\left[\ln ^{1 / 2}\left(1-\left(2 \omega N^{2} \tau_{1 i}\right)^{1 / 2}+\omega N^{2} \tau_{1 i}\right]\right. \\
& \times\left[1+\left(2 \omega \tau_{1 i}\right)^{1 / 2}+\omega \tau_{1 i}\right] \\
& \times\left[1+\left(2 \omega N_{2} \tau_{1 i}\right)^{1 / 2}+\omega N^{2} \tau_{1 i}\right] \\
& \left.\times\left[1-\left(2 \omega \tau_{1 i}\right)^{1 / 2}+\omega \tau_{1 i}\right]\right\} \\
& +2\left\{\tan ^{-1}\left[1+\left(2 \omega N^{2} \tau_{1 i}\right)^{1 / 2}\right]\right. \\
& -\tan ^{-1}\left[1+\left(2 \omega \tau_{1 i}\right)^{1 / 2}\right] \\
& +\tan ^{-1}\left[\left(2 \omega N^{2} \tau_{1 i}\right)^{1 / 2}-1\right] \\
& \left.-\tan ^{-1}\left[\left(2 \omega \tau_{1 i}\right)^{1 / 2}-1\right]\right\} \\
& \left.+8 \pi /\left[a a_{0} N\left(2 \omega \xi_{0 i} / k T\right)^{1 / 2}\right]\right] \\
&
\end{aligned}
$$

with

$$
\tau_{1 i}=a_{0}^{2} \xi_{0 i} / 6 \pi^{2} k T
$$

The overall curve of $\tan \delta$ vs. T of the $\mathrm{B}$ region of a semi-crystalline polymer, $\tan \delta_{\mathrm{b}}$, can be approximated by ${ }^{6}$

$$
\tan \delta_{\mathrm{b}}=\sum_{i=1}^{p} \tan \delta_{i} \cdot G_{i}
$$

where $p$ is the number of 2 nd-order elements constituting the A region (see Figure 2), and $G_{i}$ is the elastic modulus fraction of the $i$-th 2 ndorder element given by

$$
G_{i}=m_{i} / \sum_{i=1}^{p} m_{i}
$$

Here, $m_{i}$ is the relaxation intensity of the $\alpha_{a}$ dispersion for the $i$-th 2 nd-order element.

A parameter representing the packing density of segments (or simply, the packing density) $n$ for the 2nd-order element whose peak temperature of $\tan \delta$ is $T_{\max }^{\prime}$, is defined by

$$
n=\left(T_{\max }^{\prime}-T_{\max }\right) /\left(\Delta T_{1 / 2}\right)_{R}
$$

where $T_{\max }$ is the peak temperature of the experimental $\tan \delta-T$ curve of the sample (in question) in the $\alpha_{a}$ dispersion region, and $\left(\Delta T_{1 / 2}\right)_{R}$, the half-value width of the $\tan \delta-T$ curve of the 2 nd-order element. This value corresponds to the half value width of $\tan \delta-T$ curve for the sample constituted by the polymer chains in homogeneous packing. The $n$ value of the $i$-th 2 nd-order element, $n_{i}$, is calculated by eq 13

$$
n_{i}=\left(T_{\max i}^{\prime}-T_{\max }\right) /\left(\Delta T_{1 / 2}\right)_{R}
$$

where, $T_{\max i}^{\prime}$ is the peak temperature of $\tan \delta$ of the $i$-th 2 nd-order element. The aggregation state of $n=0$ indicates the mean aggregation state of the sample, and $n>0$ indicates a more densely aggregated state polymer segments than the mean state. When the value of $n$ of the 2nd-order element is one, the free volume fraction of this element is less than the mean free volume fraction by about $1 \%{ }^{7}$ When $n_{i}$ distributes continuously over a wide range, the modulus fraction $G_{i}$ is transformed to a continuous function of $n, F(n)$ as 


$$
F(n) \mathrm{d} n=\sum_{i, n<n_{i}<n+\mathrm{d} n} G_{i}
$$

Here, $\sum_{i, n<n_{i}<n+\mathrm{d} n}$ indicates the summation of the $G_{i}$ of the $i$-th 2 nd-order element whose $n_{i}$ distributes between $n$ and $n+\mathrm{d} n$. The function of $F(n)$ has the physical meaning of the distribution function of the packing density of polymer segment in an amorphous region. On substituting eq 14 into eq 10 ,

$$
\tan \delta_{\mathrm{b}}=\int_{-\infty}^{\infty} \tan \delta(n) F(n) \mathrm{d} n
$$

where $\tan \delta(n)$ indicates the $\tan \delta_{i}$ of the $i$-th 2 nd-order element whose $n_{i}$ equals $n$. When $\tan \delta_{\mathrm{b}}$ is known in advance, $F(n)$ can be obtained by solving the integral equation of eq 15. In practice, it is difficult to solve eq 15 accurately.

The numerical calculation of $\tan \delta(n)$ shows that the $\tan \delta(n) v s$. $T$ curve can be approximated as an equilateral triangle shape. ${ }^{1}$ When this shape approximation of $\tan \delta(n)$ is employed, eq 15 transforms into eq $16^{1}$

$$
\begin{aligned}
& F(n)=\left(T^{2} / T_{\max }\right)\left[1 /\left(\tan \delta_{\mathrm{b}}\right)_{\max , R}\right]\left\{\tan \delta_{\mathrm{b}} / T\right. \\
&+T \int_{T_{1}}^{T}\left(\tan \delta_{\mathrm{b}} / T^{3}\right) \mathrm{d} T \\
&+\left[T\left(\ln T_{1}-\ln T\right) /\left(\ln T_{\mathrm{h}}-\ln T_{1}\right)\right] \\
&\left.\times \int_{T_{1}}^{T_{\mathrm{h}}}\left(\tan \delta_{\mathrm{b}} / T^{3}\right) \mathrm{d} T\right\} \\
&\left(\tan \delta_{\mathrm{b}}\right)_{\max , R}=\left[-9+\left(81+240 B_{2}\right)^{1 / 2}\right] / 20(17) \\
& B_{2}=\left(T_{\max } / \Delta T_{1 / 2(s)}\right) \int_{T_{1}}^{T_{\mathrm{h}}}\left\{\tan \delta_{\mathrm{b}} / T\right. \\
&+T \int_{T_{1}}^{T}\left(\tan \delta_{\mathrm{b}} / T^{3}\right) \mathrm{d} T \\
&+\left[T\left(\ln T_{1}-\ln T\right) /\left(\ln T_{\mathrm{h}}-\ln T_{1}\right)\right] \\
&\left.\times \int_{T_{1}}^{T_{\mathrm{h}}}\left(\tan \delta_{\mathrm{b}} / T^{3}\right) \mathrm{d} T\right\} \mathrm{d} T
\end{aligned}
$$

$$
\Delta T_{1 / 2(s)}=5.24 \times 10^{-3} T_{\max }^{2} / \Delta H_{a}
$$

where, $T_{\mathrm{h}}$ and $T_{1}$ are the upper and lower temperatures, respectively, when $\tan \delta$ becomes zero in the $\alpha_{a}$ dispersion range. $\left(\Delta T_{1 / 2}\right)_{R}$ in eq 12 is given by the approximation equations obtained by the numerical calculation of eq 8 as follow

$\left(\Delta T_{1 / 2}\right)_{R}=\Delta T_{1 / 2(s)}\left[5(\tan \delta)_{\max , R} / 3+1.5\right]$

The value of $(\tan \delta)_{\max , R}$ corresponds to the peak value of the imaginary $\tan \delta-T$ curve calculated using the RTA theory, assuming the fine structure of the amorphous region that is the aggregation of the segments to be homogeneous. $\Delta T_{1 / 2(s)}$ is the half value width of $\tan \delta-T$ curve of the system with a single relaxation time. ${ }^{7}$ The ratio of the total value of the elastic modulus (i.e., relaxation intensity) relating to the $\alpha_{a}$ dispersion to the modulus before the $\alpha_{a}$ dispersion for the $\mathrm{B}$ region, $f_{\mathrm{eb}}$, is given as a function of $\left(\tan \delta_{\mathrm{b}}\right)_{\max , R}$

$$
\begin{aligned}
f_{\mathrm{eb}}= & 74.4\left(\tan \delta_{\mathrm{b}}\right)_{\max , R} \\
& \div\left\{\left[9.07 /\left(\tan \delta_{\mathrm{b}}\right)_{\max , R}+0.244\right)\right. \\
& \left.-7.37]+74.4\left(\tan \delta_{\mathrm{b}}\right)_{\max , R}\right\}
\end{aligned}
$$

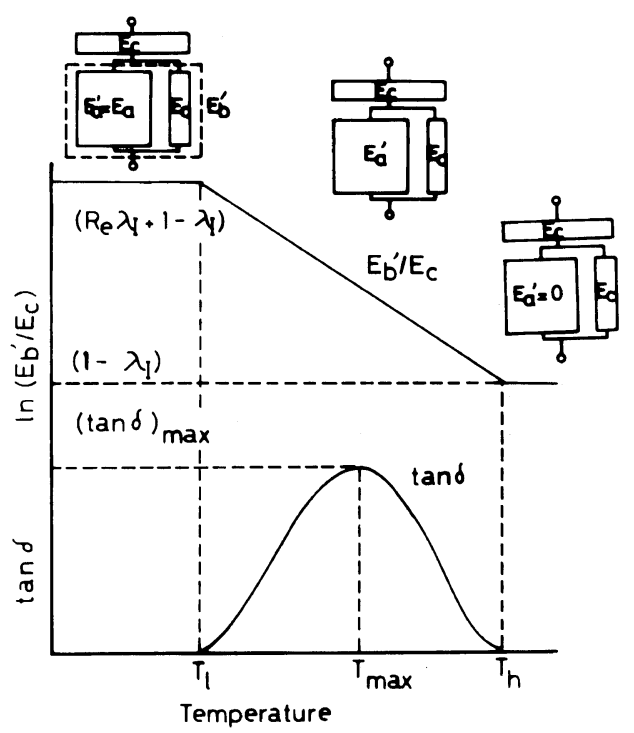

Figure 4. Dynamically equivalent model (model I) and temperature dependences of $\ln \left(E_{\mathrm{b}}{ }^{\prime} / E_{\mathrm{c}}\right)$ and $\tan \delta$.

Polymer J., Vol. 16, No. 5, 1984 
By substituting experimental values of $\Delta H_{a}$ and $T_{\max }$ into eq $19, \Delta T_{1 / 2(s)}$ can be calculated. When we place the values of $\tan \delta_{\mathrm{b}}$ in the temperature range between $T_{1}$ and $T_{\mathrm{h}}$ into eq 18, we obtain $B_{2}$ and then $\left(\tan \delta_{\mathrm{b}}\right)_{\max , R}$. After numerical calculation of eq 16 using $\left(\tan \delta_{\mathrm{b}}\right)_{\max , R}$ thus obtained and $\tan \delta_{\mathrm{b}}$, we get $F(n)$. The heterogeneity index $n_{t}$ is defined as $(\tan \delta)_{\max , R} /(\tan \delta)_{\max }$ and represents the heterogeneity of segment packing in an amorphous region. ${ }^{1}$ Here, $(\tan \delta)_{\max }$ is the peak value of $\tan \delta$ for the entire system (see Figure 4).

\section{Calculation of $\operatorname{Tan} \delta_{\mathrm{b}}$ from the Tan $\delta$ of the \\ Whole System}

The viscoelastic properties of an actual semi-crystalline polymer solid, with the fine structure shown in Figure 1a, can be better represented by Takayanagi's model (Figure 1b). Tan $\delta$ of model I in Figure $3 \mathrm{~b}$ for the whole polymer solid in the $\alpha_{a}$ dispersion region can be theoretically expressed in terms of $\tan \delta_{\mathrm{b}}$ and the dynamic moduli of the $\mathrm{B}$ and $\mathrm{C}$ regions, $E_{\mathrm{b}}{ }^{\prime}$ and $E_{\mathrm{c}}{ }^{\prime}$ as

$$
\begin{aligned}
\tan \delta= & {\left[\left(\chi_{a} / \lambda_{\mathrm{I}}\right) \tan \delta_{\mathrm{b}}\right] /\left[\left(1-\chi_{a} / \lambda_{\mathrm{I}}\right)\right.} \\
& \left.\times\left(E_{\mathrm{b}}{ }^{\prime} / E_{\mathrm{c}}{ }^{\prime}\right)\left(1-\tan ^{2} \delta_{\mathrm{b}}\right)+\chi_{a} / \lambda_{\mathrm{I}}\right]
\end{aligned}
$$

If the ratio $E_{\mathrm{b}}{ }^{\prime} / E_{\mathrm{c}}{ }^{\prime}, \chi_{a}$, and $\lambda_{\mathrm{I}}$ are known in advance, we can calculate $\tan \delta_{\mathrm{b}}$ from $\tan \delta$, using eq 22. For this purpose, we assume that

(1) $\tan \delta=0$ at $T>T_{\mathrm{h}}$ and $T<T_{1}$

(2) $E_{\mathrm{c}}{ }^{\prime}=E_{\mathrm{c}}$ at $T<T_{\mathrm{h}}\left(E_{\mathrm{c}}\right.$ is the elastic modulus of $\mathrm{C}$ region at $T=T_{1}$ )

$$
E_{\mathrm{b}}{ }^{\prime} / E_{\mathrm{c}}{ }^{\prime}=\left(R_{\mathrm{e}}-1\right) \lambda_{\mathrm{I}}+1 \quad \text { at } \quad T<T_{1}
$$

with $R_{\mathrm{e}}=E_{\mathrm{a}} / E_{\mathrm{c}}\left(E_{\mathrm{a}}\right.$ is the elastic modulus of $\mathrm{A}$ region at $T=T_{1}$ )

$$
\begin{aligned}
\ln \left(E_{\mathrm{b}}{ }^{\prime} / E_{\mathrm{c}}{ }^{\prime}\right)= & \ln \left[R_{\mathrm{e}} \lambda_{\mathrm{I}}+\left(1-\lambda_{\mathrm{I}}\right)\right] \\
& -\left(T-T_{1}\right) /\left(T_{\mathrm{h}}-T_{1}\right) \\
& \times \ln \left[R_{\mathrm{e}} \lambda_{\mathrm{I}}+\left(1-\lambda_{\mathrm{I}}\right)\right] /\left(1-\lambda_{\mathrm{I}}\right) \\
& \text { at } \quad T_{1} \leqq T \leqq T_{\mathrm{h}}
\end{aligned}
$$

$$
E_{\mathrm{b}}{ }^{\prime} / E_{\mathrm{c}}{ }^{\prime}=1-\lambda_{\mathrm{l}} \quad \text { at } \quad T>T_{\mathrm{h}}
$$

$R_{\mathrm{e}}$ has the physical meaning of the ratio between the elastic modulus of an amorphous region showing no $\alpha_{a}$ dispersion and one of a crystalline region.

Figure 4 represents schematically the above assumptions. Appendix II gives the derivation of eq $25 . E_{\mathrm{b}}{ }^{\prime} / E_{\mathrm{c}}{ }^{\prime}$ at $T_{1} \leqq T \leqq T_{\mathrm{h}}$ can be calculated from $\lambda_{\mathrm{I}}$ and $R_{\mathrm{e}}$. Consequently, we can calculate $\tan \delta_{\mathrm{b}}$ from $\tan \delta$ when $\lambda_{\mathrm{I}}$ and $R_{\mathrm{e}}$ are determined by the method given below. If we can evaluate all structural parameters such as the orientation function of crystal axis and the molecular chain axis in an amorphous region as well as all tensor values for the elastic constants of crystalline and amorphous regions, $R_{\mathrm{e}}$ may be represented theoretically using theory of solid elasticity in principle. Since the calculation of $R_{\mathrm{e}}$ is too complicated practically, it is useless to establish a complete theoretical equation for $R_{\mathrm{e}}$ when analyzing $\tan \delta-T$ curve. Thus, $R_{\mathrm{e}}$ should be determined by trial and error, with the aid of a computer.

Figure 5 shows a block diagram for determining $R_{\mathrm{e}}$ and $\lambda_{\mathrm{I}}$ using the experimental $\tan \delta-T$ curve. The ratio between the relaxation intensity of the $\alpha_{a}$ dispersion and modulus before the $\alpha_{a}$ dispersion is given by $f_{\mathrm{e}} \cdot f_{\mathrm{e}}$ also has the meaning of the elastic modulus fraction of segments contributing to the $\alpha_{a}$ dispersion. $f_{\mathrm{e}}$ is evaluated by application of the MKN method to the $\tan \delta-T$ curve of the whole system. But $f_{\mathrm{e}}$ of model I shown in Figure $3 \mathrm{~b}, f_{\mathrm{el}}$, is given by

$f_{\mathrm{eI}}=R_{\mathrm{e}} \chi_{a} /\left[R_{\mathrm{e}} \lambda_{\mathrm{I}}+\left(1-\lambda_{\mathrm{I}}\right)\right]\left(1-\lambda_{\mathrm{I}}+\chi_{a}\right)$

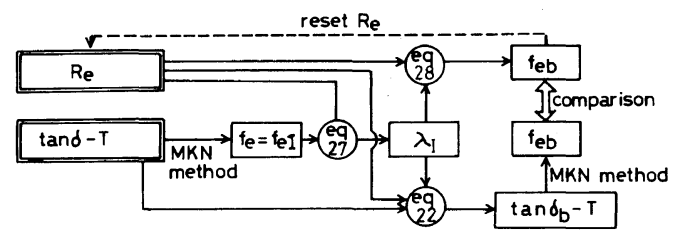

Figure 5. Block diagram for determination of $R_{\mathrm{e}}$ and $\lambda_{\mathrm{I}}$ using the experimental $\tan \delta-T$ curve. 
$f_{\mathrm{e}}$ for the whole system should be independent of dynamically equivalent models adopted for numerical calculation. Consequently, the equation, $f_{\mathrm{el}}=f_{\mathrm{e}}$ holds. Assuming $R_{\mathrm{e}}$ to be $R_{\mathrm{e} 1}$, $\lambda_{\mathrm{I}}$ can be calculated by substituting $R_{\mathrm{e} 1}$ into eq 27. The $\lambda_{\mathrm{I}}$ thus obtained is denoted as $\lambda_{\mathrm{I} 1}$. On substituting both values of $R_{\mathrm{e} 1}$ and $\lambda_{\mathrm{I} 1}$ into eq 25 and both values of $E_{\mathrm{b}}{ }^{\prime} / E_{\mathrm{c}}{ }^{\prime}$ given by eq 25 and $\lambda_{\mathrm{I} 1}$ into eq 22 , we can calculate $\tan \delta_{\mathrm{b}}$ from $\tan \delta$. We calculate $f_{\mathrm{eb}}$ by applying the MKN method to $\tan \delta_{\mathrm{b}}$ thus obtained. The definition of $f_{\mathrm{eb}}$, i.e., $f_{\mathrm{e}}$ for the $\mathrm{B}$ region, leads to the following equation

$$
f_{\mathrm{eb}}=R_{\mathrm{e}} \lambda_{\mathrm{I}} /\left[R_{\mathrm{e}} \lambda_{\mathrm{I}}+\left(1-\lambda_{\mathrm{I}}\right)\right]
$$

Putting the values of $f_{\mathrm{eb}}$ and $\lambda_{11}$ into eq 28 , we obtain the value for $R_{\mathrm{e}}$. We denote this $R_{\mathrm{e}}$ value as $R_{\mathrm{e} 2}$. If $R_{\mathrm{e} 1}$ is set in an appropriate range, the difference between $R_{\mathrm{e} 1}$ and $R_{\mathrm{e} 2}$ becomes negligible. But if we set $R_{\mathrm{e} 1}$ so as to make the difference between $R_{\mathrm{e} 1}$ and $R_{\mathrm{e} 2}$ negligibly small, we may conclude $R_{\mathrm{e} 1}$ to be reasonable. Reasonable values of $\lambda_{\mathrm{I}}$ and $\tan \delta_{\mathrm{b}}$ are calculated by this $R_{\mathrm{e} 1}$ using the same procedure for determining $\lambda_{\mathrm{I} 1}$ and $\tan \delta_{\mathrm{b}}$ as mentioned above.

\section{Method for Analyzing Tan $\delta-T$ curve: $M K$ Method}

The analysis procedure of $\tan \delta-T$ curve is summarized in Figure 6. The theoretical background of this procedure has already been presented. The double tetragonal frame indicates the observed value and the tetragonal frame surrounded by the broken line, the analysis procedure of the MKN method. The practical analyzing procedure is given as follows:

(1) The observed values needed $\operatorname{are} \tan \delta$, $\Delta H_{\dot{a}}$, and $\chi_{\dot{a}}$.

(2) The value of $f_{\mathrm{e}}$ is calculated by applying the MKN method to the $\tan \delta$ - $T$ curve.

(3) The $R_{\mathrm{e}}$ value is set to an appropriate value of $R_{\mathrm{e} 1}$.

(4) On putting the value of $f_{\mathrm{e}}\left(f_{\mathrm{e}}=f_{\mathrm{el}}\right)$ obtained in the procedure (2), $\chi_{a}$, and $R_{\mathrm{e} 1}$ into

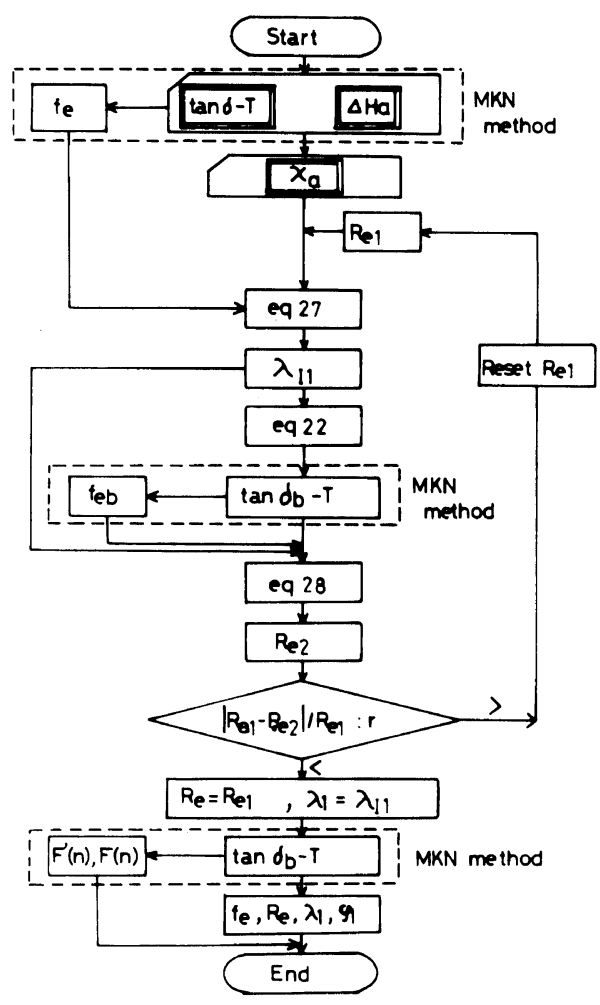

Figure 6. Flow chart for analysis of $\tan \delta$ vs. the temperature curve (MK method).

eq $27, \lambda_{\mathrm{I} 1}$ is calculated.

(5) Tan $\delta_{\mathrm{b}}-T$ curve is calculated using eq 22,25 , and also the values of $\tan \delta, R_{\mathrm{e} 1}, \chi_{a}$, and $\lambda_{\mathrm{I} 1}$.

(6) By applying the MKN method to $\tan \delta_{\mathrm{b}}-T$ curve, we obtain $f_{\mathrm{eb}}$.

(7) The $R_{\mathrm{e}}$ value (denoted by $R_{\mathrm{e} 2}$ ) is calculated by putting $f_{\mathrm{eb}}$ and $\lambda_{\mathrm{I} 1}$ into eq 28 .

(8) We reset $R_{\mathrm{e}}\left(R_{\mathrm{e} 1}\right)$ until the following relation is satisfied

$$
\left|R_{\mathrm{e} 1}-R_{\mathrm{e} 2}\right| / R_{\mathrm{e} 1}>r
$$

where, $r$ is the relative error which we can set arbitrarily.

(9) The $R_{\mathrm{e} 1}$ satisfying eq 29 can be regarded as a reasonable value for $R_{\mathrm{e}} . \lambda_{\mathrm{I}}$ and $\tan \delta-T$ curve are then calculated by following steps (3) to (5).

(10) $F(n)$ is evaluated by applying the 
MKN method to the $\tan \delta_{\mathrm{b}}-T$ curve. $F^{\prime}(n)$, a normalized distribution function of the packing density of the whole sample, is calculated by the following equation

$$
F^{\prime}(n)=f_{\mathrm{e}} F(n)
$$

\section{EXPERIMENTAL}

\section{Sample Preparation}

Nylon 66 chips (viscosity-average molecular weight determined using the molecular weightintrinsic viscosity number relation $7 ; 1.8 \times 10^{4}$, amino end group content; 52 meq kg ${ }^{-1}$, carboxyl end group content; 70 meq $\mathrm{kg}^{-1}$ ) manufactured by Asahi Chemical Ind. Co. Ltd. were employed for preparing the undrawn fiber. Spinning was carried out by using an extruder with a $15 \mathrm{~mm}$ diameter; the ratio of the length to diameter was $25: 1$. The extruder was equipped with three 100-mesh screen nets and the die had a hole $1 \mathrm{~mm}$ in diameter. The lengthdiameter ratio was $8: 1$ and the entrance angle, $90^{\circ}$. The temperature of the die during spinning was $265^{\circ} \mathrm{C}$ and the linear velocity of the polymer melt at the outlet of the die was $1.52-4.76 \mathrm{~m} \mathrm{~min}^{-1}$. The take up speed was $47-59 \mathrm{~m} \mathrm{~min}^{-1}$. The birefringence of the undrawn fiber at $25^{\circ} \mathrm{C}$ under green light (wave length; $546 \mathrm{~nm}$ ) ranged from $3.56 \times 10^{-3}$ to $6.67 \times 10^{-3}$. The oriented fibers were prepared by cold drawing the above undrawn fibers at $25^{\circ} \mathrm{C}$ at a drawing speed of $300 \% / \mathrm{min}$. The commercially obtained Nylon 66 fibers (referred to as NAF), manufactured by Asahi Chemical Ind. Co., Ltd. were also used.

\section{Measurement}

The isochronal and isothermal dynamic viscoelasticities were measured using a Rheovibron DDV-II manufactured by ToyoBoldwin Co., Ltd. (Japan). The measuring frequency in the isochronal measurement was $110 \mathrm{~Hz}$ and the heating rate, $2^{\circ} \mathrm{C} \mathrm{min}^{-1}$ in dry air. When making the isothermal measurement, the temperature was controlled at a given temperature to within $0.2^{\circ} \mathrm{C}$ in dry air. The temperature range of the isothermal measurement was from 25 to $160^{\circ} \mathrm{C}$, and the frequency range, from 0.01 to $110 \mathrm{~Hz}$.

\section{RESULTS AND DISCUSSION}

\section{Validity of Approximation Equation 25}

The dynamic modulus $E^{\prime}$ of the model I $\left(=E_{\mathrm{I}}{ }^{\prime}\right)$, is given by

$E_{\mathrm{I}}{ }^{\prime}=\left(E_{\mathrm{b}}{ }^{\prime} / E_{\mathrm{c}}{ }^{\prime}\right)\left[\chi_{a} / \lambda_{\mathrm{I}}+\left(1-\chi_{a} / \lambda_{\mathrm{I}}\right) E_{\mathrm{b}}{ }^{\prime} / E_{\mathrm{c}}{ }^{\prime}\right]$

If eq 25 represents $E_{\mathrm{b}}{ }^{\prime} / E_{\mathrm{c}}{ }^{\prime}$ precisely, the observed dynamic modulus should coincide with $E_{\mathrm{I}}^{\prime}$ in eq 31 over the temperature range, $T_{1}$ to $T_{\mathrm{h}}$.

Figure 7 compares $E_{\mathrm{I}}^{\prime}$ (broken line) and the observed $E^{\prime}$ (open mark) for the Nylon 66 fibers. The coincidence between both $E^{\prime}$ is fairly good and thus, the reliability of eq 25 and model $\mathrm{I}$ is ensured.

\section{Comparison of $M K$ and MKN Methods}

The accuracy of $R_{\mathrm{e}}$ determined by the MK method should be checked in advance for setting the relative error $r$ in eq 29. The theoretical values of $E_{\mathrm{c}}$ reported are distrib-

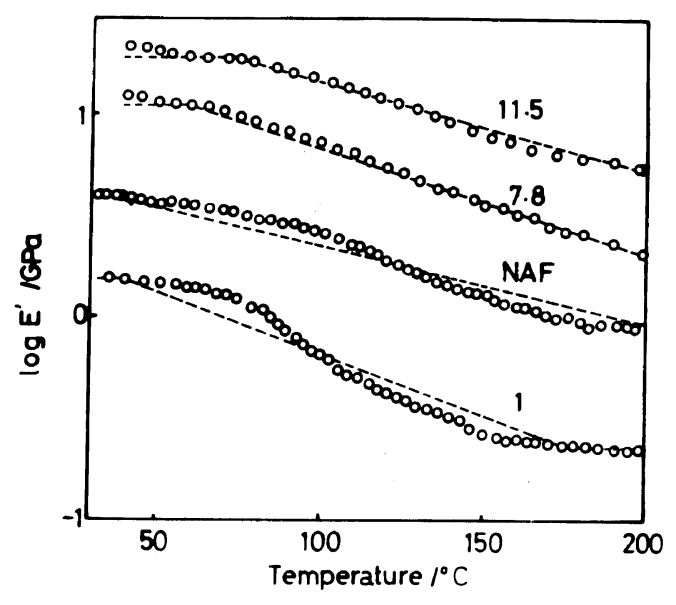

Figure 7. $\log E^{\prime}$ vs. $T$ curves for various Nylon 66 fibers: Numbers indicate draw ratios, NAF stands for the commercially obtained Nylon 66 fibers, broken lines, calculated values using eq 24,25 , and 26 , open marks, observed values. 


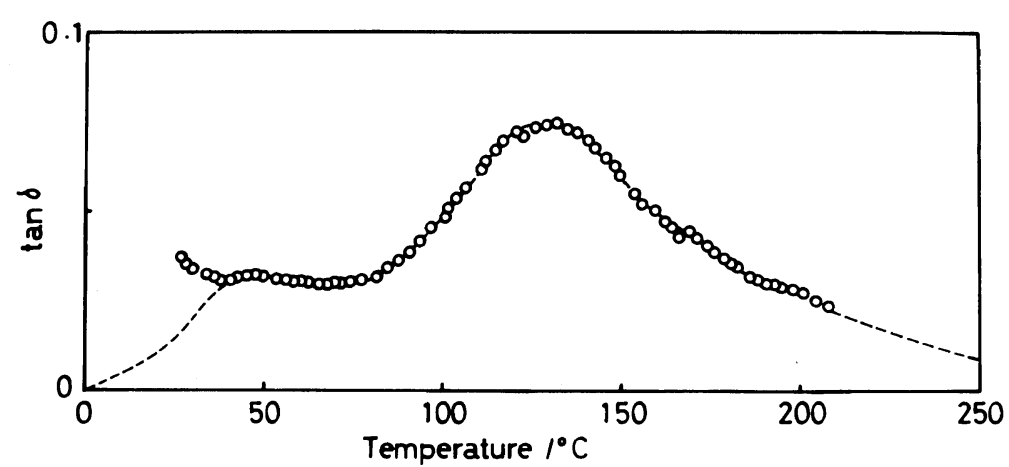

Figure 8. Tan $\delta$ vs. $T$ curve for NAF in the temperature region of the $\alpha_{a}$ absorption: Broken line, curves utilized in the actual analysis; Open marks, observed values.

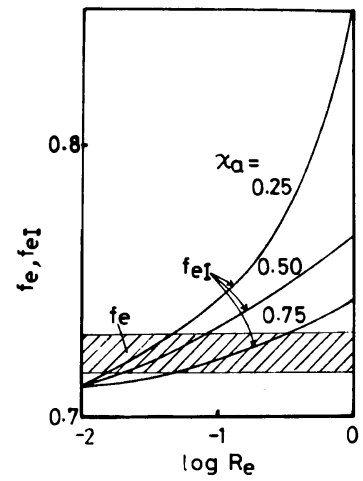

Figure 9. $R_{\mathrm{e}}$ dependence of $f_{\mathrm{el}}$ of NAF for various $\chi_{a}$ values: The numbers indicate $\chi_{a}$ values, hatched region; the region of $f_{\mathrm{el}}$ of $0.724 \pm 0.007$.

uted between 1 and $100 \mathrm{GPa}^{8} E_{\mathrm{a}}$ at $T<T_{1}$ is about $1 \mathrm{GPa}$, as estimated from the van der Waals interaction force. The theoretical $R_{\mathrm{e}}$ is thus in the range from 0.01 to 1.0 .

Figure 8 shows the observed $\tan \delta-T$ curve for NAF in the temperature range of the $\alpha_{a}$ dispersion. We can read $T_{1}$ of $273 \mathrm{~K}, T_{\mathrm{h}}$ of $543 \mathrm{~K}$, the peak value of $\tan \delta,(\tan \delta)_{\max }$, of 0.075 , and the peak temperature of $\tan \delta, T_{\max }$, of $404 \mathrm{~K}$. The apparent activation energy $\Delta H_{a}$ of $55 \mathrm{kcal} \mathrm{mol}^{-1}$ was determined experimentally.

Figure 9 shows the $R_{\mathrm{e}}$ dependence of $f_{\mathrm{eI}}$ for NAF calculated by eq 27 using the given values of $\chi_{a}$ in this figure. $f_{\mathrm{e}}$ obtained by the MKN method for the whole sample was $0.724 \pm 0.007$ (hatched region in Figure 9). The dependence of $f_{\mathrm{eI}}$ on $\log R_{\mathrm{e}}$ (represented by $\left.\partial f_{\mathrm{el}} / \partial \log R_{\mathrm{e}}\right)$ increased with a decrease in $\chi_{a}$ and with an increase in $R_{\mathrm{e}}$. The $\chi_{a}$ dependence of $f_{\mathrm{eI}}$ (represented by $\partial f_{\mathrm{el}} / \partial \chi_{a}$ ) increased with increasing $R_{\mathrm{e}}$. When the relative error of $f_{\mathrm{eI}}$ was $1 \%, R_{\mathrm{e}}$ determined by the MK method distributed between 0.043 and 0.015 for $\chi_{a}=$ 0.25 , between 0.090 and 0.020 for $\chi_{a}=0.5$, and between 0.32 and 0.056 for $\chi_{a}=0.75$ even if $r$ in eq 29 was less than 0.01 .

Assuming $\chi_{a}$ to be $\left(1-\chi_{\mathrm{c}}\right)$, we get an $\chi_{a}$ value of 0.6 for NAF. $R_{\mathrm{e}}$ from Figure 9 distributes from 0.16 to 0.032 when $r=0.01$, and is within the theoretical $R_{\mathrm{e}}$ value. In a practical case, since there are some uncertainties in $\chi_{a}$ when evaluating it, the $R_{\mathrm{e}}$ obtained will also contain these uncertainties.

Figure 10 shows the changes in $\tan \delta_{\mathrm{b}}-T$ curves when $R_{\mathrm{e}}$ goes from 0.01 to 1.0 at $\chi_{a}=$ 0.25 (Figure 10a), 0.5 (Figure 10b), and 0.75 (Figure 10c). The deviation in $\tan \delta_{\mathrm{b}}$ from $\tan \delta$ accompanied with change in $R_{\mathrm{e}}$ becomes appreciable with small $\chi_{a}$. When $R_{\mathrm{e}}$ increases at constant $\chi_{a}$, this deviation becomes large. The difference between $\tan \delta_{\mathrm{b}}$ and $\tan \delta$ causes the differences in the results of the MK and MKN methods, and thus, the difference in $F(n)$ between these methods cannot be ignored with large $R_{\mathrm{e}}$ and small $\chi_{a}$. When $\chi_{a}$ is less than $50 \%$ at $R_{\mathrm{e}}>0.1$, the MK method should be used in preference. $F_{\mathrm{b}}{ }^{\prime}(n)$, defined as the distribution function of the packing density of polymer 

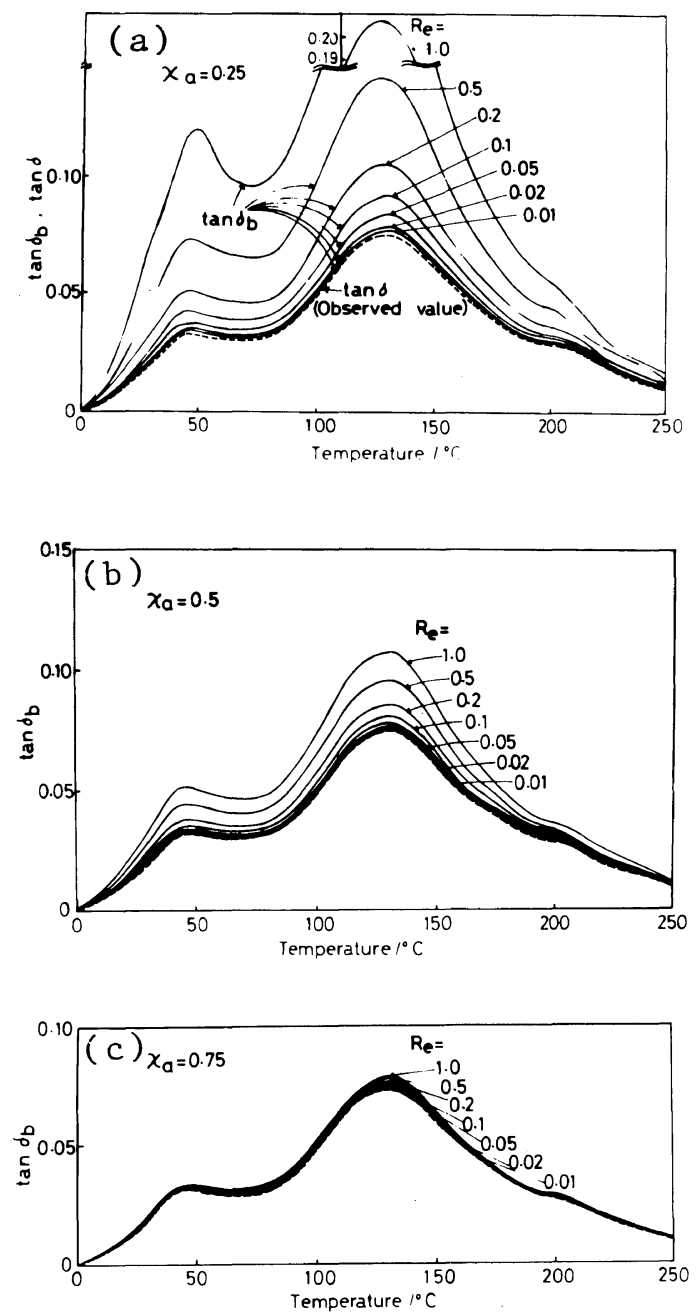

Figure 10. Tan $\delta_{\mathrm{b}}$ vs. $T$ curve for various values of $R_{\mathrm{e}}$ : (a), $\chi_{a}=0.25$; (b), $\chi_{a}=0.50$; (c), $\chi_{a}=0.75$; broken line, observed $\tan \delta$ vs. $T$ curve for NAF.

segment normalized in the $\mathrm{B}$ region, is given by the following equation:

$$
F_{\mathrm{b}}{ }^{\prime}(n)=f_{\mathrm{eb}} F(n)
$$

The $F_{\mathrm{b}}{ }^{\prime}(n)-n$ curves thus obtained at various $R_{\mathrm{e}}$ are shown in Figure 11. This figure also shows the results obtained by applying the MKN method to the $\tan \delta-T$ curve, $F_{m}{ }^{\prime}(n)$ (broken line). When $\chi_{a}$ is small, the $F_{\mathrm{b}}{ }^{\prime}(n)-n$ curves change markedly with change in $R_{\mathrm{e}}$. When $R_{\mathrm{e}}$ increases, the peak value of $F_{\mathrm{b}}{ }^{\prime}(n)$
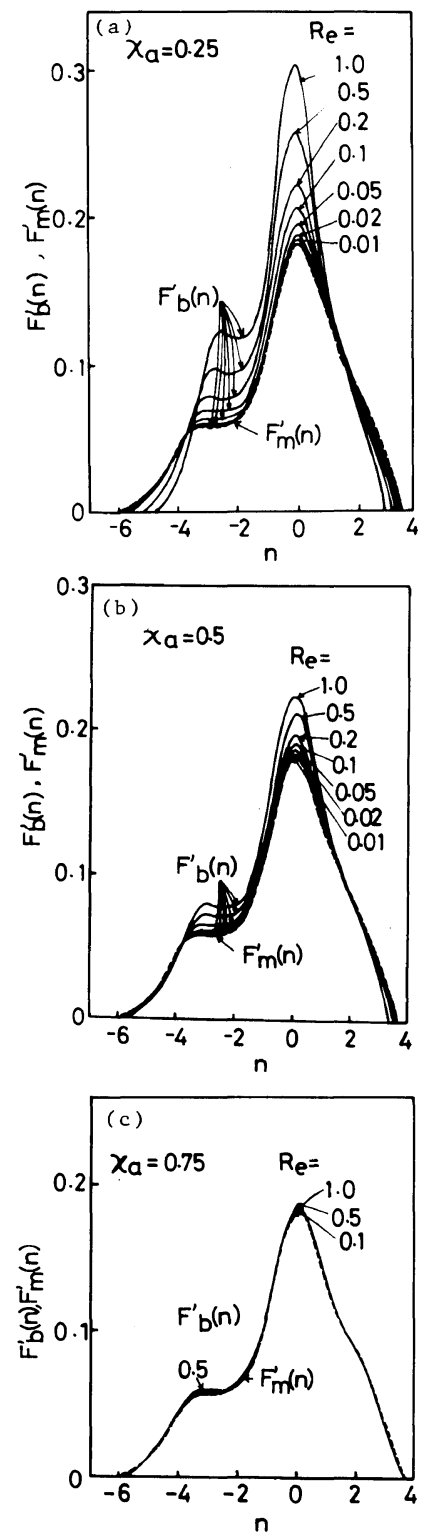

Figure 11. $F^{\prime}{ }_{\mathrm{b}}(n)$ vs. $n$ curve for various values of $R_{\mathrm{e}}$ : (a), $\chi_{a}=0.25$; (b), $\chi_{a}=0.50$; (c), $\chi_{a}=0.75$; broken line, $F_{\mathrm{m}}{ }^{\prime}(n)$ obtained by using the MKN method.

becomes large and the shape of the $F_{\mathrm{b}}{ }^{\prime}(n)-n$ curve sharpens and the peak position of $F_{\mathrm{b}}{ }^{\prime}(n)$ shifts to a smaller $n$ value. The mean value of $n, \bar{n}$ (defined by $\left.\int_{0}^{\infty} n F_{\mathrm{b}}{ }^{\prime}(n) \mathrm{d} n / \int_{0}^{\infty} F_{\mathrm{b}}{ }^{\prime}(n) \mathrm{d} n\right)$ also becomes small. When $\chi_{a}$ is 0.25 and $R_{\mathrm{e}} 0.1$, the heterogeneity index $n_{t}$ obtained by the MKN 

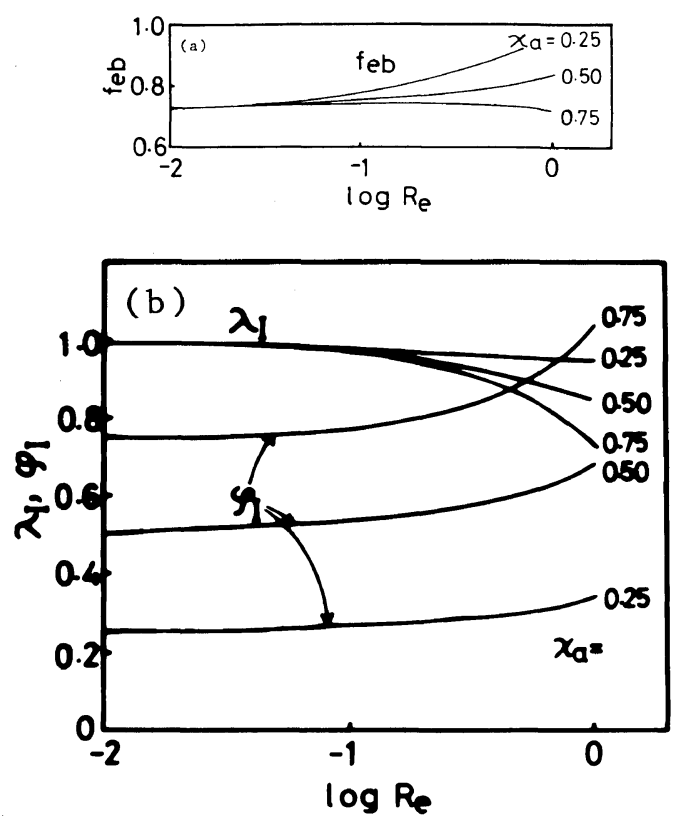

Figure 12. $R_{\mathrm{e}}$ dependences of $f_{\mathrm{eb}}, \lambda_{\mathrm{I}}$ and $\phi_{\mathrm{I}}$ for various values of $\chi_{a}$ : (a), $f_{\mathrm{eb}} ;(\mathrm{b}), \lambda_{\mathrm{I}}$ and $\phi_{\mathrm{I}}$; the figures indicate the values of $\chi_{a}$.

method $^{1}$ is overestimated by about $4 \%$, and $\bar{n}$ shifts to a larger value of about 0.1 , compared to that of the MK method.

Figure 12 shows the $R_{\mathrm{e}}$ dependences of $f_{\mathrm{eb}}$, $\lambda_{\mathrm{I}}$, and $\phi_{\mathrm{I}}$. In the range $R_{\mathrm{e}}<0.1, f_{\mathrm{eb}}$ and $\lambda_{\mathrm{I}}$ increase slightly and $n_{t \mathrm{~b}}$ and $\lambda_{\mathrm{I}}$ decrease slightly with an increase in $R_{\mathrm{e}}$. But when $R_{\mathrm{e}}$ is larger than $0.1, f_{\mathrm{eb}}, \lambda_{\mathrm{I}}$, and $\phi_{\mathrm{I}}$ show marked dependence on $\chi_{a}$ and $R_{\mathrm{e}}$. Consequently, the accuracy of $R_{\mathrm{e}}$ may determine the reliability of the values for $F^{\prime}(n)$, and $f_{\mathrm{eb}}$ obtained by the MK method when $R_{\mathrm{e}}>0.1$.

The results of the numerical calculations mentioned above indicate that only when $\left(1-\chi_{a}\right) R_{\mathrm{e}}<0.02$ is the MKN method useful, provided the relative error of $F^{\prime}(n)$ is less than $10 \%$.

Application of the MK Method to Nylon 66 Fibers

In the case of Nylon 66 fibers, $\chi_{a}$ is 0.4 and $R_{\mathrm{e}} 0.1$. Thus, $\left(1-\chi_{a}\right) R_{\mathrm{e}}$ is larger than 0.02 . Thus, the MK method should be used. Figure

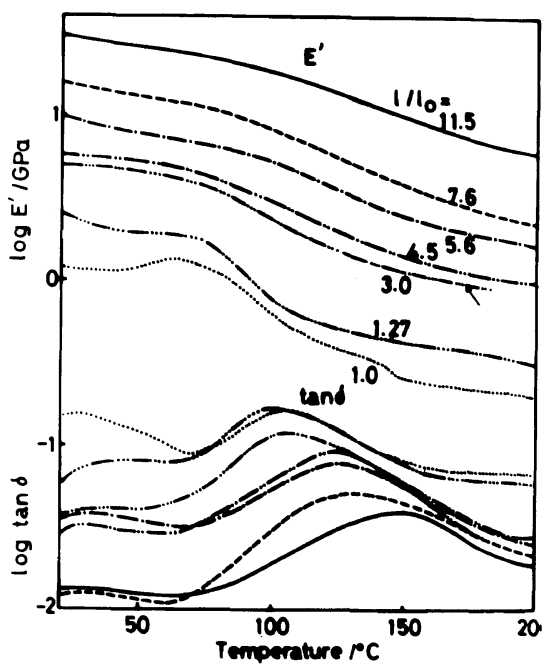

Figure 13. Temperature dependences of $E^{\prime}$ and $\tan \delta$ for the Nylon 66 fibers: The figures indicate draw ratios.

13 shows the temperature dependence of $E^{\prime}$ and $\tan \delta$ observed. By applying the MK method to the data in Figure 13 at $R_{\mathrm{e}}=0.1$ and $r=0.01$, we obtain the parameters of $\lambda_{\mathrm{I}}$ and $\lambda_{\text {II }}$ representing the dispersion state of crystalline and amorphous regions, the elastic modulus fractions $f_{\mathrm{eb}}$ and $f_{\mathrm{em}}$ (the suffix of $\mathrm{m}$ indicates the results obtained by the MKN method), having the same value of $f_{\mathrm{e}}$ in eq 30. The standard deviations of $F^{\prime}(n)$ and $F_{\mathrm{m}}{ }^{\prime}(n), \sigma$ and $\sigma_{\mathrm{m}} \cdot \lambda_{\mathrm{II}}$ are calculated by eq 1 from $\lambda_{\mathrm{I}}$ and $\chi_{a}$. The data thus obtained appear in Figure 14. Both methods show similar draw ratio dependences for all characteristic values in Figure 14. Both $\lambda_{\mathrm{I}}$ and $f_{\mathrm{eb}}$ are maximum at a draw ratio between 1 and 3 . Thus, at the initial stage of drawing, the series type connection of crystalline and amorphous regions along the draw direction developes and beyond this point, the parallel type connection increases in its relative fraction of various connection types. The maxima of $n_{\mathrm{t}}$ not shown and $\sigma$ near a draw ratio of 3 indicates that the heterogeneity of the packing state of polymer molecules in the amorphous region increases at the initial stage of drawing, and then decreases with an increase in the 

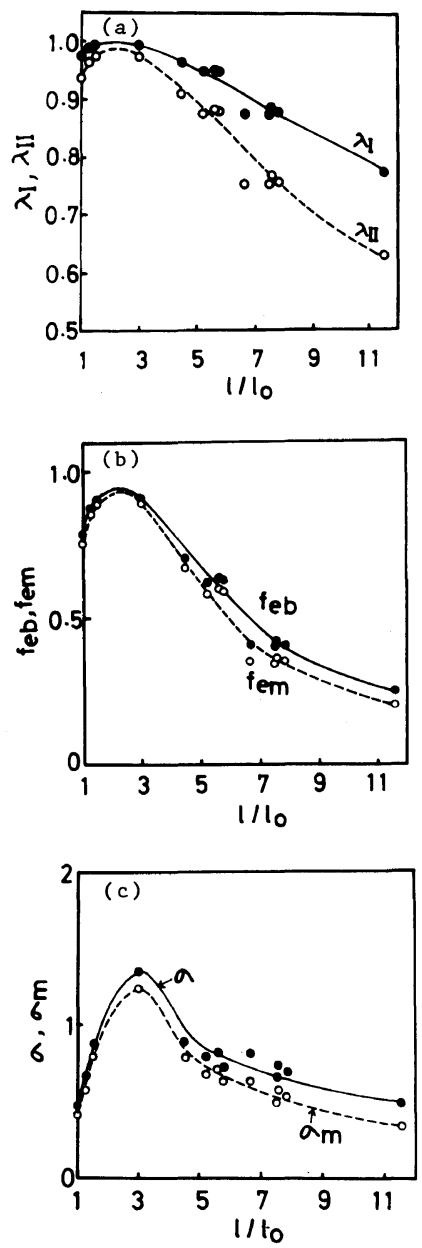

Figure 14. Draw ratio dependences of $\lambda_{\mathrm{I}}, \lambda_{\mathrm{II}}, f_{\mathrm{em}}, f_{\mathrm{eb}}$, $\sigma_{\mathrm{m}}$, and $\sigma$ for the Nylon 66 fibers: (a), $\lambda_{\mathrm{I}}$ (closed mark) and $\lambda_{\text {II }}$ (open mark); (b), $f_{\text {em }}$ (open mark) and $f_{\text {eb }}$ (closed mark); (c), $\sigma_{\mathrm{m}}$ (open mark) and $\sigma$ (closed mark).

draw ratio until the tensile fractures.

The curves of $F_{\mathrm{b}}{ }^{\prime}(n)$ (and also $F^{\prime}(n)$ ) vs. $n$ and $F_{m}{ }^{\prime}(n) v s . n$ for the Nylon 66 fibers with various draw ratios are shown in Figure 15. With an increase in the draw ratio, the $F_{\mathrm{b}}{ }^{\prime}(n)$ component at small $n$ decreases drastically. These changes in $F_{\mathrm{b}}{ }^{\prime}(n)$ with draw ratio cause the peak location of $F_{\mathrm{b}}{ }^{\prime}(n)$ to shift to large $n$. The $n$ value where $F_{\mathrm{b}}{ }^{\prime}(n)$ equals zero increases slightly with the draw ratio. That is, the packing state of polymer molecules.in the amorphous region takes on a more dense state.
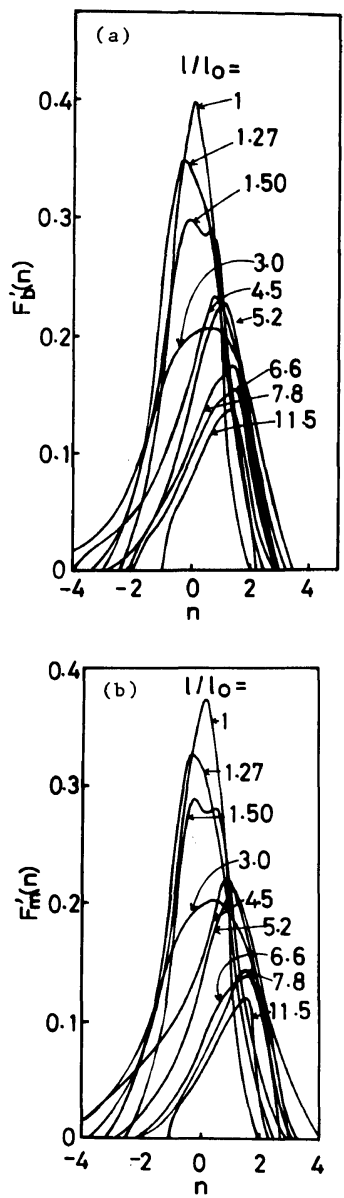

Figure 15. $F^{\prime}(n)$ vs. $n$ curves for the Nylon 66 fibers with different draw ratios: $(\mathrm{a}), F_{\mathrm{b}}{ }^{\prime}(n)$ (full line); (b), $F_{\mathrm{m}}{ }^{\prime}(n)$; the figures indicate draw ratios.

\section{CONCLUSION}

By the MK method, the following can be obtained from the experimental $\tan \delta$ vs. $T$ curve, $\Delta H_{a}$ and $\chi_{\mathrm{c}}\left(=1-\chi_{a}\right)$ : (1) $\lambda_{\mathrm{I}}$ and $\phi_{\mathrm{I}}$ representing the amorphous components acting in parallel and in series for an external force, (2) a quantitative representation of the fine structure of the amorphous region no longer influenced by the heterogeneity of the mixing state of the crystal and non-crystal regions, and (3) the elastic modulus ratio $R_{\mathrm{e}}$. None of this information can be obtained by the MKN method. 
The differences in various quantities such as the heterogeneity index determined by these methods, are within $10 \%$ of their relative values provided $\left(1-\chi_{a}\right) R_{\mathrm{e}}$ is less than 0.02 .

Acknowledgement. The authors are grateful to Professor M. Takayanagi for his helpful comments and suggestions throughout the course of this work.

\section{REFERENCES}

1. S. Manabe, K. Kamide, C. Nakayama, and S. Kobayashi, J. Text. Mach. Soc. Jpn., 30, T85 (1977); and also J. Text. Mach. Soc. Jpn., English Edition, 27, 10 (1981).

2. P. E. Rouse, J. Chem. Phys., 21, 1272 (1963).

3. A. V. Tobolsky and J. J. Aklonis, J. Phys. Chem., 68, 1970 (1964).

4. S. Manabe and K. Kamide, J. Text. Mach. Soc. Jpn., 34, T93 (1981).

5. M. Takayanagi, Mem. Fac. Eng. Kyushu Univ., 23, 41 (1963).

6. For example, M. Takayanagi, IUPAC-Pure Appl. Chem., 23, 151 (1970).

7. K. Kamide and S. Manabe, Sen-i Gakkaishi, 34, P-70 (1978).

8. K. Kamide, A. Kataoka, and T. Kawai, Makromol. Chem., 139, 221 (1970).

9. For example, I. Sakurada, T. Ito, and K. Nakamae, J. Polym. Sci., 15, C75 (1966).

10. D. Kaplan and N. W. Tschoegl, Polym. Eng. Sci., 14, 43 (1974).

11. M. L. Williams and J. D. Ferry, J. Polym. Sci., 11, 169 (1953).

12. T. L. Smith, Trans. Soc. Rheol., 2, 131 (1958).

\section{APPENDIX I}

\section{Derivation of $E q 1$}

The complex moduli of model I and model II ( $E_{\mathrm{I}}^{*}$ and $E_{\mathrm{II}}^{*}$, respectively) can be expressed using the moduli of the amorphous and crystalline regions $\left(E_{\mathrm{a}}{ }^{*}\right.$ and $\left.E_{\mathrm{c}}{ }^{*}\right)$ as follows:

$$
\begin{aligned}
E_{\mathrm{I}}{ }^{*}= & \left\{\phi_{\mathrm{I}} /\left[E_{\mathrm{a}}{ }^{*} \lambda_{\mathrm{I}}+E_{\mathrm{c}}{ }^{*}\left(1-\lambda_{\mathrm{I}}\right)\right]\right. \\
& \left.+\left(1-\phi_{\mathrm{I}}\right) / E_{\mathrm{c}}{ }^{*}\right\}^{-1} \\
E_{\mathrm{II}}^{*}= & \left(1-\lambda_{\mathrm{II}}\right) E_{\mathrm{c}}{ }^{*} \\
& +\lambda_{\mathrm{II}} /\left[\phi_{\mathrm{II}} / E_{\mathrm{a}}{ }^{*}+\left(1-\phi_{\mathrm{II}}\right) / E_{\mathrm{c}}{ }^{*}\right]
\end{aligned}
$$

The physical meanings of $\lambda$ and $\phi$ give the relations of eq A-I.3 and A-I.4,

$$
\begin{aligned}
& \lambda_{\mathrm{I}} \phi_{\mathrm{I}}=\chi_{a} \\
& \lambda_{\mathrm{II}} \phi_{\mathrm{II}}=\chi_{a}
\end{aligned}
$$

Since the complex moduli of model I and II are the same,

$$
E_{\mathrm{I}}^{*}=E_{\mathrm{II}}^{*}
$$

Putting $\phi_{\mathrm{I}}$ of eq A-I.3 into eq A-I.1 and $\phi_{\text {II }}$ of eq A-I.4 into eq A-I.2, we obtain $E_{\mathrm{I}}^{*}$ and $E_{\mathrm{II}}^{*}$ as functions of $\lambda_{\mathrm{I}}$ and $\lambda_{\mathrm{II}}$, respectively. The combination of $E_{\mathrm{I}}{ }^{*}$ and $E_{\mathrm{II}}^{*}$ and eq A-I.5 leads to

$$
\begin{aligned}
& \left\{\left(1-\lambda_{\mathrm{II}}\right)+\lambda_{\mathrm{II}} /\left[\left(E_{\mathrm{c}}{ }^{*} / E_{\mathrm{a}}^{*}\right)\left(\chi_{a} / \lambda_{\mathrm{II}}\right)\right.\right. \\
& \left.\left.\quad+\left(1-\chi_{a} / \lambda_{\mathrm{II}}\right)\right]\right\}\left\{\chi_{a} /\left[\left(E_{\mathrm{a}}{ }^{*} / E_{\mathrm{c}}{ }^{*}\right) \lambda_{\mathrm{I}}\right.\right. \\
& \left.\left.\quad+\left(1-\lambda_{\mathrm{I}}\right)\right] \lambda_{\mathrm{I}}+1-\chi_{a} / \lambda_{\mathrm{I}}\right\}=1.0
\end{aligned}
$$

By solving eq A-I.6 for $\lambda_{\mathrm{I}}$, we get eq 1 as

$$
\lambda_{\mathrm{I}}=\left[\left(1+\chi_{a}\right) \lambda_{\mathrm{II}}-\chi_{a}^{2}\right] / \lambda_{\mathrm{II}} \chi_{a}
$$

Equation 1 is equivalent to that derived by Kaplan and Tschoegl ${ }^{9}$ for the dynamic moduli of model I and model II (eq A-8 in ref 9).

\section{APPENDIX II}

\section{Derivation of $E q 25$}

Williams and Ferry ${ }^{11}$ derived the equation correlating the relaxation spectrum $H(\ln \tau)$ with the dynamic modulus $E^{\prime}$ when $m$ $(=\mathrm{d} \ln H(\ln \tau) / \mathrm{d} \ln \tau)$ is less than 1. A semicrystalline polymer solid satisfies this condition and thus the eq A-II.1 derived by them can be applied to this polymer.

$$
H(\ln \tau)=A E^{\prime}\left[\mathrm{d} \ln E^{\prime} /\left.\mathrm{d} \ln \tau\right|_{\omega \tau=1}\right]
$$

where $A=\sin (m \pi / 2) /(m \pi / 2)$ and $\omega$ is the measuring frequency. $\mathrm{Smith}^{12}$ derived the relation between $H(\ln \tau)$ and the loss modulus $E^{\prime \prime}$ as follows

$$
E^{\prime \prime}=\left.(\pi / 2) H(\ln \tau) \sec (m \pi / 2)\right|_{\omega \tau=1}
$$

By putting eq A-II.2 into eq A-II.1, we obtain $\mathrm{d} \ln E^{\prime} / \mathrm{d} \ln \tau=(2 / \pi) E^{\prime \prime} /\left[A E^{\prime} \sec (m \pi / 2)\right]$

$$
=m \tan \delta
$$


When isochronal measurement of viscoelasticity is carried out, it is more convenient to transform eq A-II. 3 into eq A-II. 4 going on the assumption that the reduced variable method for measuring frequency and temperature holds.

$$
\mathrm{d} \ln E^{\prime} / \mathrm{d} \ln \omega_{0} a_{\mathrm{T}}=m \tan \delta
$$

$\omega_{0}$ is the isochronal measuring frequency and $a_{\mathrm{T}}$, the shift factor from a reference temperature $T_{0}$ to a measuring temperature $T$. Integration of eq A-II. 4 gives

$$
\ln E^{\prime}=\ln E_{0}{ }^{\prime}+m \int_{\ln \omega_{0}}^{\ln \omega_{0}+\ln a_{\mathrm{T}}} \tan \delta \mathrm{d} \ln \omega_{0} a_{\mathrm{T}}
$$

where $E_{0}{ }^{\prime}$ is $E^{\prime}$ at $T=T_{0}$.

When eq A-II.5 is applicable to B region, eq A-II.6 holds

$$
\ln E_{\mathrm{b}}{ }^{\prime}=\ln E_{\mathrm{b}, 0}^{\prime}+m \int_{\ln \omega_{0}}^{\ln \omega_{0}+\ln a_{\mathrm{T}}} \tan \delta_{\mathrm{b}} \mathrm{d} \ln \omega_{0} a_{\mathrm{T}}
$$

where $E_{\mathrm{b}, 0}^{\prime}$ is $E_{\mathrm{b}}{ }^{\prime}$ at $T=T_{0}$. We employ $T_{1}$ as $T_{0}$. Since $E_{\mathrm{c}}{ }^{\prime}$ is independent of temperature (i.e., $\left.\quad E_{\mathrm{c}}{ }^{\prime}=E_{\mathrm{c}}\right)$ and $E_{\mathrm{b}, 0}{ }^{\prime} / E_{\mathrm{c}}=R_{\mathrm{e}} \lambda_{\mathrm{I}}+\left(1-\lambda_{\mathrm{I}}\right)$, then

$$
\begin{aligned}
\ln E_{\mathrm{b}}{ }^{\prime} / E_{\mathrm{c}}= & \ln \left[R_{\mathrm{e}} \lambda_{\mathrm{I}}+\left(1-\lambda_{\mathrm{I}}\right)\right]+\left(m / E_{\mathrm{c}}\right) \\
& \times \int_{\ln \omega_{0}}^{\ln \omega_{0}+\ln a_{\mathrm{T}}} \tan \delta_{\mathrm{b}} \mathrm{d} \ln \omega_{0} a_{\mathrm{T}}
\end{aligned}
$$

When $\tan \delta_{\mathrm{b}}$ can be approximated as a constant value $\left(\tan \delta_{\mathrm{m}}\right)$ in the temperature range of $T_{1}$ and $T$, eq A-II.7 is transformed as

$\ln E_{\mathrm{b}}{ }^{\prime} / E_{\mathrm{c}}=\ln \left[R_{\mathrm{e}} \lambda_{\mathrm{I}}+\left(1-\lambda_{\mathrm{I}}\right)\right]+k \ln a_{\mathrm{T}} \quad(\mathrm{A}-\mathrm{II} .8)$ where $k$ is $m \cdot \tan \delta_{\mathrm{m}} / E_{\mathrm{c}} \cdot k$ should be satisfied by a boundary condition such that when $T=T_{\mathrm{h}}$ then $E_{\mathrm{b}}{ }^{\prime} / E_{\mathrm{c}}=1-\lambda_{\mathrm{I}}$. As for the $\alpha_{a} \mathrm{ab}-$ sorption, $a_{\mathrm{T}}$ can be approximated by the following WLF type equation

$\ln a_{\mathrm{T}}=C_{2}\left(T-T_{1}\right) /\left[C_{1}+\left(T-T_{1}\right)\right]$

where, $C_{1}$ and $C_{2}$ are constants independent of temperature. When $T_{1}$ is $T_{\mathrm{g}}$, then eq A-II.9 coincides with WLF equation.

Substituting eq A-II.9 into eq A-II.8 and applying the boundary condition mentioned above, eq A-II.10 is derived as

$$
\begin{aligned}
k= & {\left[\left(T-T_{1}\right) /\left(T_{\mathrm{h}}-T_{1}\right)\right] } \\
& \times\left[\left(C_{1}+T_{\mathrm{h}}-T_{1}\right) /\left(C_{1}+T-T_{1}\right)\right] \\
& \left.\times \ln \left\{\left[R_{\mathrm{e}} \lambda_{\mathrm{I}}+\left(1-\lambda_{\mathrm{I}}\right)\right] /\left(1-\lambda_{\mathrm{I}}\right)\right]\right\}
\end{aligned}
$$

In the WLF equation (i.e., when $T_{1}=T_{\mathrm{g}}$ ) $C_{1}$ can be approximated to 51.6 and $\left(C_{1}+T_{\mathrm{h}}-T_{1}\right) /\left(C_{1}+T-T_{1}\right)$ may thus be regarded as unity. Therefore, eq A-II.10 reduces to eq 25

$$
\begin{aligned}
\ln \left(E_{\mathrm{b}}{ }^{\prime} / E_{\mathrm{c}}\right)= & \ln \left[R_{\mathrm{e}} \lambda_{\mathrm{I}}+\left(1-\lambda_{\mathrm{I}}\right)\right] \\
& -\left[\left(T-T_{1}\right) /\left(T_{\mathrm{h}}-T_{1}\right)\right] \\
& \times \ln \left\{\left[R_{\mathrm{e}} \lambda_{\mathrm{I}}+\left(1-\lambda_{\mathrm{I}}\right)\right] /\left(1-\lambda_{\mathrm{I}}\right)\right\}
\end{aligned}
$$

